\title{
On Weak Projection Invariant Semisimple Modules
}

\author{
Ramazan Yaşar \\ Hacettepe-ASO 1.OSB Vocational School, Hacettepe University, Ankara, Türkiye
}

\author{
Article Info \\ Keywords: Exchange property, Extend- \\ ing module, Projection invariant sub- \\ module \\ 2010 AMS: 16D10, 16D80 \\ Received: 28 January 2021 \\ Accepted: 05 April 2021 \\ Available online: 27 May 2021
}

\begin{abstract}
We introduce and investigate the notion of weak projection invariant semisimple modules. We deal with the structural properties of this new class of modules. In this trend we have indecomposable decompositions of the special class of the former class of modules via some module theoretical properties. As a consequence, we obtain when the finite exchange property implies full exchange property for the latter class of modules.
\end{abstract}

\section{Introduction}

All rings are associative with unity and modules are unital right modules. Let $R$ be a ring and $M$ a right $R$-module. Recall that $M$ is called $C S$ (or, extending) if every submodule of $M$ is essentially contained in a direct summand of $M$. This kind of modules are important generalizations of injective, semisimple and uniform modules. There have been several generalizations of $C S$ modules as well as some classes of modules which are related to the direct summands of the module in literature (see [1]-[4]).

A submodule $N$ of $M$ is called projection invariant, if $f(N) \subseteq N$ for all $f^{2}=f \in E n d\left(M_{R}\right)$ (see [3, 5, 6]). Note that torsion subgroup of a group, socle of a module and the radical of a ring are all projection invariant submodules of the corresponding modules, respectively. Recall from [6], a module $M$ is called $\pi$-extending if every projection invariant submodule of $M$ is essential in a direct summand of $M$. It is well-known that a $C S$-module is $\pi$-extending [3].

In this paper, we introduce and investigate the notion of weak projection invariant semisimple modules which is a generalization of semisimple and projection invariant semisimple modules [7]. We call a module $M$ is weak projection invariant semisimple, denoted by $w \pi$-semisimple, provided that each semisimple projection invariant submodule of $M$ is a direct summand of $M$. It is clear that the class of the $w \pi$-semisimple modules is contained in the class of $\pi$-extending modules. We deal with structural module properties of $w \pi$-semisimple modules. Moreover, we define special class of $w \pi$-semisimple modules and obtain indecomposable decomposition for the aforementioned modules via Abelian endomorphism rings over rings with ascending chain condition on the right annihilators. As a consequence, we obtain that the finite exchange property implies full exchange property.

Let $X \subseteq M$, then $X \leq M$, SocM and $E n d\left(M_{R}\right)$ denote $X$ is a submodule of $M$, the socle of $M$ and the endomorphism ring of $M_{R}$, respectively. Recall that a module $M$ over a ring $R$ is said to have (finite) exchange property if for any (finite) index set $I$, whenever $M \oplus Y=\bigoplus_{i \in I} A_{i}$ for modules $Y$ and $A_{i}$, then $M \oplus Y=M \oplus\left(\oplus_{i \in I} B_{i}\right)$ for submodules $B_{i}$ of $A_{i}$ [8]. A family $\left\{N_{i}: i \in I\right\}$ of independent submodules of a module $M$ is said to be a local summand if for any finite subset $F$ of $I, \underset{i \in F}{\oplus} N_{i}$ is a direct summand of $M[3,9]$. Recall further that a ring $R$ is called Abelian if every idempotent of $R$ is central [3, 10]. 
Since $w \pi$-semisimple modules are based on semisimple projection invariant submodules, we start with the following basic result.

Lemma 1.1. (i) If $A$ is projection invariant in $B$ and $B$ is projection invariant in $M$ then $A$ is projection invariant in $M$. (ii) If $M=\bigoplus_{i \in I} M_{i}$, and $X$ is a semisimple projection invariant submodule of $M$, then $X=\underset{i \in I}{\oplus}\left(X \cap M_{i}\right)$ and $X \cap M_{i}$ is semisimple projection invariant submodule of $M_{i}$ for all $i \in I$.

Proof. Immediate by definitions (see [11, p.50]).

In [7, Lemma 1.2], the author attempts to obtain the following statement: Let $M_{R}$ be a module and $N \leq K \leq M_{R}$. If $N$ is projection invariant in $M$ and $K / N$ is projection invariant in $M / N$, then $K$ is projection invariant in $M$. However, the proof therein is inconsistent. Since $f(N)$ would be nonzero, the function $\theta: M / N \rightarrow M, \theta(m+N)=f(m)$ for all $m \in M$ is not well-defined. Let us make it clear by the following example.

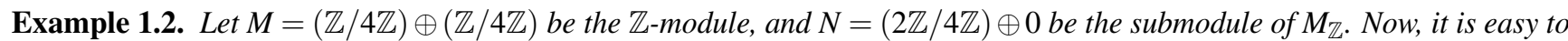
see that $\operatorname{End}\left(M_{\mathbb{Z}}\right) \cong\left[\begin{array}{ll}\mathbb{Z} / 4 \mathbb{Z} & \mathbb{Z} / 4 \mathbb{Z} \\ \mathbb{Z} / 4 \mathbb{Z} & \mathbb{Z} / 4 \mathbb{Z}\end{array}\right]$. Let $f^{2}=f=\left[\begin{array}{ll}\overline{1} & \overline{1} \\ \overline{0} & \overline{0}\end{array}\right] \in \operatorname{End}\left(M_{\mathbb{Z}}\right)$. So, let us consider $\theta: M / N \rightarrow M, \theta(m+N)=f(m)$ for all $m \in$ M. Since $(\overline{1}+\overline{3})-(\overline{3}+\overline{3}) \in N,(\overline{1}+\overline{3})+N=(\overline{3}+\overline{3})+N$. But $\theta((\overline{1}+\overline{3})+N)=f(\overline{1}+\overline{3})=\left[\begin{array}{ll}\overline{1} & \overline{1} \\ \overline{0} & \overline{0}\end{array}\right]\left[\begin{array}{l}\overline{1} \\ \overline{3}\end{array}\right]=\left[\begin{array}{l}\overline{0} \\ \overline{0}\end{array}\right]$, and $\theta((\overline{3}+\overline{3})+N)=f(\overline{3}+\overline{3})=\left[\begin{array}{ll}\overline{1} & \overline{1} \\ \overline{0} & \overline{0}\end{array}\right]\left[\begin{array}{l}\overline{3} \\ \overline{3}\end{array}\right]=\left[\begin{array}{l}\overline{2} \\ \overline{0}\end{array}\right]$. Hence $\theta((\overline{1}+\overline{3})+N) \neq \theta((\overline{3}+\overline{3})+N)$.

Notice that Proposition 2.3 (ii), Corollaries 2.4, 2.5 and one part of the proof of Theorem 2.6 in [7] use [7, Lemma 1.2]. By the previous example, the aforementioned results are also invalid.

\section{Main results}

In this section, we introduce and investigate the class of weak projection invariant semisimple modules. We focus on some structural properties of weak projection invariant semisimple modules as well as indecomposable decompositions for the special class of the weak projection invariant semisimple modules via some module theoretical conditions.

Definition 2.1. We call an $R$-module $M$ weak projection invariant semisimple, denoted by w $\pi$-semisimple, if each semisimple projection invariant submodule of $M$ is a direct summand of $M$.

Observe that any semisimple module and $\pi$-semisimple module is $w \pi$-semisimple. Moreover, any module which has zero socle (for example, a polynomial ring $R[x]$ over any ring $R$ ) is clearly a $w \pi$-semisimple module. Next, we provide $w \pi$-semisimple modules which are not $\pi$-semisimple.

Example 2.2. (i) Let $M$ be the $\mathbb{Z}$-module $\mathbb{Z}$. Obviously, $M_{\mathbb{Z}}$ is $w \pi$-semisimple. However, $M_{\mathbb{Z}}$ is not $\pi$-semisimple. For example, $N=2 \mathbb{Z}$ is a projection invariant in $M_{\mathbb{Z}}$ which is not a direct summand of $M_{\mathbb{Z}}$.

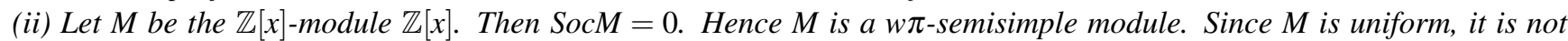
$\pi$-semisimple.

(iii) [4, Example 2.4(ii)]. Let D be a simple domain which is not a division ring. Take $R=\left[\begin{array}{cc}D & D \oplus D \\ 0 & D\end{array}\right]$ then $I=\left[\begin{array}{ll}0 & 0 \oplus D \\ 0 & 0\end{array}\right]$ is an ideal of $R$. Thus, I is a projection invariant submodule of $R_{R}$ which is not a direct summand of $R_{R}$. It follows that $R_{R}$ is not $\pi$-semisimple. However, $\operatorname{Soc}\left(R_{R}\right)=0$, and hence $R_{R}$ is $w \pi$-semisimple.

Example 2.2 sheds light on the natural question, namely, when a $w \pi$-semisimple module is a $\pi$-semisimple. The second part of the following result provides an answer.

Proposition 2.3. (i) Assume that $M_{R}$ is an indecomposable module. Then $M_{R}$ is semisimple if and only if $M_{R}$ is $w \pi$-semisimple and SocM is essential in $M$.

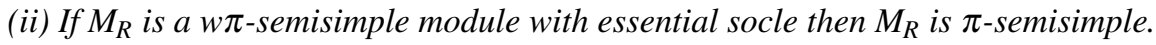

Proof. (i) $(\Rightarrow)$ This implication is clear.

$(\Leftarrow)$ Let $X \leq M$. Since $M$ is indecomposable, $X$ is projection invariant in $M$. It follows that $\operatorname{Soc} X$ is projection invariant in $M$, by Lemma 1.1 (i). By hypothesis, $\operatorname{Soc} X$ is a direct summand of $M$. Hence $\operatorname{Soc} X=0$ or $\operatorname{Soc} X=M$. Therefore $X=0$ or $M$. Thus, $X$ is a direct summand of $M$. So, $M$ is semisimple.

(ii) Let $X$ be any projection invariant submodule of $M_{R}$. Then $\operatorname{Soc} X$ is projection invariant in $M$, by Lemma 1.1 (i). It follows that $\operatorname{Soc} X$ is a direct summand of $M$. On the other hand,

$$
\operatorname{Soc} X=X \cap \operatorname{Soc} M \leq X \cap M=X
$$

gives that $\operatorname{Soc} X$ is essential in $X$. Thus $\operatorname{Soc} X=X$ i.e., $X$ is a direct summand of $M$. So, $M_{R}$ is $\pi$-semisimple. 
Corollary 2.4. If $M_{R}$ is a $w \pi$-semisimple module with essential socle then $M_{R}$ is $\pi$-extending.

Proof. Let $X$ be a projection invariant submodule of $M$. By Proposition 2.3, $X$ is a direct summand of $M$. Since $X$ is essential in itself, $M_{R}$ is a $\pi$-extending module.

Lemma 2.5. Let $M_{R}$ be $w \pi$-semisimple and $N$ a projection invariant submodule of $M$. Then $N$ is $w \pi$-semisimple.

Proof. Let $X$ be any semisimple projection invariant submodule of $N$. By Lemma 1.1 (i), $X$ is projection invariant in $M$. Therefore $M=X \oplus X^{\prime}$ for some $X^{\prime}$ submodule of $M$. Now, by Lemma 1.1 (ii), $N=(N \cap X) \oplus\left(N \cap X^{\prime}\right)=X \oplus\left(N \cap X^{\prime}\right)$. Thus $X$ is a direct summand of $N$ which yields that $N$ is $w \pi$-semisimple.

Lemma 2.6. Let $M_{R}$ be a $w \pi$-semisimple module. Then the following statements hold:

(i) Every fully invariant submodule of $M_{R}$ is $w \pi$-semisimple.

(ii) If End $\left(M_{R}\right)$ is Abelian then every direct summand of $M_{R}$ is $w \pi$-semisimple.

Proof. (i) Since every fully invariant submodule is projection invariant, the proof follows from Lemma 2.5.

(ii) Let $M_{R}$ be a $w \pi$-semisimple module with an Abelian endomorphism ring. Let $K=e M$ for some $e^{2}=e \in E n d\left(M_{R}\right)$. Thus $g(e M) \subseteq e M$ for all $g^{2}=g \in E n d\left(M_{R}\right)$. Hence $K_{R}$ is a projection invariant submodule of $M_{R}$. By Lemma $2.5, K_{R}$ is a $w \pi$-semisimple module.

Proposition 2.7. Let $M=M_{1} \oplus M_{2}$ such that $M_{2}$ is semisimple fully invariant submodule of $M$. If $M_{R}$ is $w \pi$-semisimple, then both $M_{1}$ and $M_{2}$ are $w \pi$-semisimple.

Proof. It is clear that $M_{2}$ is $w \pi$-semisimple. Let $X$ be a semisimple projection invariant submodule of $M_{1}$. Then $X \oplus M_{2}$ is a semisimple projection invariant submodule of $M$ (see [6, Lemma 4.13]). By hypothesis, $X \oplus M_{2}$ is a direct summand of $M$. Hence $M=X \oplus M_{2} \oplus L$ for some submodule $L$ of $M$. Now, the modular law gives that

$$
M_{1}=M_{1} \cap\left(X \oplus M_{2} \oplus L\right)=X \oplus\left(M_{1} \cap\left(M_{2} \oplus L\right)\right) .
$$

Hence $X$ is a direct summand of $M_{1}$ which yields that $M_{1}$ is $w \pi$-semisimple.

Theorem 2.8. Let $M=\underset{i \in I}{\oplus} M_{i}$ where $M_{i}$ 's are fully invariant submodules of $M$ for $i \in I$. If $M_{i}$ is $w \pi$-semisimple for all $i \in I$, then $M$ is $w \pi$-semisimple.

Proof. Assume each $M_{i}$ is $w \pi$-semisimple for all $i \in I$ and $M=\underset{i \in I}{\oplus} M_{i}$. Let $N$ be a semisimple projection invariant submodule of $M$. Then $N=\bigoplus_{i \in I}\left(N \cap M_{i}\right)$ where $N \cap M_{i}$ is a semisimple projection invariant submodule of $M_{i}$ for all $i \in I$, from Lemma 1.1(ii). By assumption, $M_{i}$ is $w \pi$-semisimple which gives that $N \cap M_{i}$ is a direct summand of $M_{i}$ for all $i \in I$. It follows that $N$ is a direct summand of $M$. Thus, $M$ is $w \pi$-semisimple.

Observe that if $\mathrm{M}$ is a $w \pi$-semisimple module in the previous result then by Lemma 2.5 , each $M_{i}$ is also $w \pi$-semisimple for all $i \in I$. Our next aim is to obtain an indecomposable decomposition for special $w \pi$-semisimple modules. To do this, let us give the following definition.

Definition 2.9. We call an $R$-module $M w \pi^{*}$-semisimple provided that whenever any semisimple projection invariant submodule is contained as projection invariant in a projection invariant submodule of $M$ then the larger submodule is a direct summand of $M$.

It can be seen easily that any $w \pi^{*}$-semisimple module is $w \pi$-semisimple (any projection invariant submodule has a semisimple projection invariant submodule, namely, its socle). However, there are several $w \pi$-semisimple modules which are not $w \pi^{*}$-semisimple. For example, let $M$ be the $\mathbb{Z}$-module $\mathbb{Z}$ (see, Example 2.2 (i)).

Lemma 2.10. Let $R$ be a ring and $M$ an $R$-module such that $R$ satisfies ascending chain condition on right annihilators of the form $r(m)(m \in M)$. If $M$ is $w \pi^{*}$-semisimple with an Abelian endomorphism ring then $M$ has an indecomposable decomposition.

Proof. Let $\left\{X_{\lambda}: \lambda \in I\right\}$ be an independent family of submodules of $M$ and $X=\underset{\lambda \in I}{\oplus} X_{\lambda}$ be a local summand of $M$. Now, let us define the canonical projection $\pi_{k}: X \rightarrow \underset{k \in I, k \neq \lambda}{\oplus} X_{k}$. Then $f(X)=f\left(\underset{\lambda \in I}{\oplus} X_{\lambda}\right)=\underset{\lambda \in I}{\bigoplus} f\left(X_{\lambda}\right)=\underset{\lambda \in I}{\oplus} f\left(\right.$ ker $\left.\pi_{\lambda}\right)$ where $f^{2}=f \in$ $\operatorname{End}\left(M_{R}\right)$. By the assumption that $\operatorname{End}\left(M_{R}\right)$ is Abelian, $f\left(k e r \pi_{\lambda}\right) \subseteq \operatorname{ker} \pi_{\lambda}$. Thus $f(X) \subseteq X$. It follows that $X$ is projection invariant in $M_{R}$. Since $S o c X$ is projection invariant in $X$, by $w \pi^{*}$-semisimple, $X$ is a direct summand of $M$. Hence [9, Theorem 2.17] yields that $M$ has an indecomposable decomposition.

Next, we have the following result. 
Theorem 2.11. Let $R$ be $a$ ring and $M$ an $R$-module such that $R$ satisfies ascending chain condition on right annihilators of the form $r(m)(m \in M)$. If $M$ is $w \pi^{*}$-semisimple with an Abelian endomorphism ring then $M$ is a direct sum of uniform submodules.

Proof. Observe that being $w \pi^{*}$-semisimple implies $\pi$-extending, and an indecomposable module is uniform by [6, Proposition 3.8]. Now, we conclude the proof by Lemma 2.10 and Lemma 2.6 (ii).

It would be worthy construct an example which shows that being Abelian endomorphism ring in the previous theorem is not superfluous. Incidentally, let us give the example.

Example 2.12. Let $R$ be the real field and $n$ be any odd integer with $n \geq 1$. Let $S$ be the polynomial ring $R\left[x_{1}, \ldots, x_{n}\right]$ indeterminates $x_{1}, x_{2}, \ldots, x_{n}$ over $R$. Let $R$ be the ring $S / S s$, where $s=x_{1}^{2}+x_{2}^{2}+\cdots+x_{n}^{2}-1$. Then the free $R$-module $M=\bigoplus_{i=1}^{n} R$ contains a submodule $K_{R}$ which is indecomposable and has uniform dimension $n-1$ (see [12, Corollary 16]).

Note that $K_{R}$ is not uniform. Since SocM $=0$, then $\operatorname{Soc}\left(K_{R}\right)=0$. Now, let $Y=\left[\begin{array}{ll}S & K \\ 0 & R\end{array}\right]$ be the split null extension ring where $S=\operatorname{End}\left(K_{R}\right)$. Observe that ${ }_{S} K$ is faithful. Therefore Soc $\left(Y_{Y}\right)=0$. Hence $Y$ is a w $\pi^{*}$-semisimple module. Moreover, $Y=\left[\begin{array}{cc}S & K \\ 0 & 0\end{array}\right] \oplus\left[\begin{array}{ll}0 & 0 \\ 0 & R\end{array}\right]$ and $\left[\begin{array}{cc}S & K \\ 0 & 0\end{array}\right]$ is indecomposable with uniform dimension $n-1$. It follows that $Y$ has no decomposition into uniform submodules. It can be seen that $Y_{Y}$ is Noetherian. However, End $\left(Y_{Y}\right) \cong Y$ is not Abelian. For, let a $=\left[\begin{array}{ll}f & x \\ 0 & 0\end{array}\right]$, and $b=\left[\begin{array}{ll}0 & 0 \\ 0 & 1\end{array}\right]$ be two elements of End $\left(Y_{Y}\right)$ where $f \in S$ and $0 \neq x \in K_{R}$. Then $a b=\left[\begin{array}{ll}f & x \\ 0 & 0\end{array}\right]\left[\begin{array}{ll}0 & 0 \\ 0 & 1\end{array}\right]=\left[\begin{array}{ll}0 & x \\ 0 & 0\end{array}\right]$ and $b a=\left[\begin{array}{ll}0 & 0 \\ 0 & 1\end{array}\right]\left[\begin{array}{ll}f & x \\ 0 & 0\end{array}\right]=\left[\begin{array}{ll}0 & 0 \\ 0 & 0\end{array}\right]$

Now, we have the following consequences of the Theorem 2.11. The first one is the result on exchange property of modules which was pointed out in the introduction and the last is based on locally Noetherian modules. Recall that a module is called locally Noetherian provided that every finitely generated submodule is Noetherian (see [3]).

Corollary 2.13. Let $R$ be a right Noetherian ring and $M$ an $R$-module with an Abelian endomorphism ring. If $M$ is $w \pi^{*}-$ semisimple then the finite exchange property implies full exchange property.

Proof. By Theorem 2.11 and [8, Corollary 6].

Corollary 2.14. Let $M$ be a locally Noetherian module with an Abelian endomorphism ring. If $M$ is w $\pi^{*}$-semisimple then the finite exchange property implies full exchange property.

Proof. Let $m \in M$. Then $R / r(m) \cong m R$ is right Noetherian module. It follows that $R$ satisfies ascending chain condition on right annihilators of the form $r(m)(m \in M)$. Thus Theorem 2.11 gives the result.

Finally, we have the next result on endomorphism ring of a $w \pi^{*}$-semisimple module. First, recall that a ring $R$ is $\pi$-Baer if the right annihilator of a projection invariant left ideal of $R$ is of the form $e R$ for some $e^{2}=e \in R$ (see [5, 13]).

Theorem 2.15. Assume that $M$ is a $w \pi^{*}$-semisimple module. Then the endomorphism ring of $M$ is a $\pi$-Baer ring.

Proof. Let $S=\operatorname{End}\left(M_{R}\right)$ and $I$ be a projection invariant left ideal of $S$. We want to show that $r_{S}(I)=e S$ for some $e^{2}=e \in S$. It can be seen that $r_{M}(I)$ is a projection invariant submodule of $M_{R}$. Hence $\operatorname{Soc}\left(r_{M}(I)\right)$ is a projection invariant submodule of $r_{M}(I)$. By hypothesis, $r_{M}(I)=e M$ for some $e^{2}=e \in S$. Thus $I e M=0$, so $I e=0$, as ${ }_{S} M$ faithful. Therefore $e S \subseteq r_{S}(I)$. Now, let $a \in r_{S}(I)$. Hence $I a=0$ which gives that $l(a M)=0$. It follows that $a M \subseteq r_{M}(I)=e M$. Thus $a \in e S$, so $r_{S}(I) \subset e S$. Therefore, $\mathrm{S}$ is a $\pi$-Baer ring.

Corollary 2.16. If $M$ is a $\pi$-semisimple module then the endomorphism ring of $M$ is a $\pi$-Baer ring.

Proof. Since $\pi$-semisimple implies $w \pi^{*}$-semisimple the result follows from Theorem 2.15 .

\section{Acknowledgements}

The authors would like to express their sincere thanks to the editor and the anonymous reviewers for their helpful comments and suggestions.

\section{Funding}

There is no funding for this work. 


\section{Availability of data and materials}

Not applicable.

\section{Competing interests}

The authors declare that they have no competing interests.

\section{Author's contributions}

All authors contributed equally to the writing of this paper. All authors read and approved the final manuscript.

\section{References}

[1] N. V. Dung, D. V. Huynh, P. F. Smith, R. Wisbauer, Extending Modules, Longman, Harlow, 1994.

[2] F. T. Mutlu, On matrix rings with the SIP and the Ads, Turk. J. Math., 42 (2018), 2657 - 2663.

[3] A. Tercan, C. C. Yücel, Module theory extending modules and generalizations, Birkhauser, Basel, 2016.

[4] R. Yaşar, Modules in which semisimple fully invariant submodules are essential in summands, Turk. J. Math., 43(5) (2019), 2327-2336.

[5] G. F. Birkenmeier, Y. Kara, A. Tercan, $\pi$-Baer rings, J. Algebra App., 17(2) (2018), 1850029.

[6] G. F. Birkenmeier, A. Tercan, C. C. Yücel, The extending condition relative to sets of submodules, Comm. Algebra, 42 (2014), $764-778$.

[7] Y. Kara, On projective invariant semisimple submodules, Al-Qadisiyah J. Pure Sci., 26(1) (2020), 13-19.

[8] B. Zimmermann, W. Zimmermann, Classes of modules with the exchange property, J. Algebra, 88(2) (1984), $416-434$.

[9] S. H. Mohamed, B. J. Müller, Continuous and Discrete Modules, Cambridge University Press, 1990.

[10] G. F. Birkenmeier, J. K. Park, S. T. Rizvi, Extensions of rings and modules, Birkhauser, New York, NY, USA, 2013.

[11] L. Fuchs, Infinite Abelian Groups I, Academic Press, New York, NY, USA, 1970.

[12] A. Tercan, Weak $\left(C_{11}\right)$ modules and algebraic topology type examples, Rocky Mount J. Math., 34(2) (2004), $783-792$.

[13] I. Kaplansky, Rings of Operators, Benjamin, New York, NY, USA, 1968. 